\title{
Construction, installation and operation of ProtoDUNE-SP
}

\author{
Stefania Bordoni ${ }^{a, b, 1, *}$ \\ ${ }^{a} C E R N$, \\ European Centre for Nuclear Research, Geneva, Switzerland \\ ${ }^{b}$ Department of Physics and Astronomy, Michigan State University, MI 48824, United States \\ E-mail: stefania.bordoni@cern.ch
}

\begin{abstract}
The single-phase liquid argon TPC at CERN (ProtoDUNE-SP) is an engineering prototype for the first module of the DUNE far detector. This prototype, which has dimensions of a cube of about $10 \mathrm{~m}$ edge, provides full validation of the use of the membrane tank technology for large dimension cryostats. Furthermore, the very high performance of the protoDUNE-SP TPC with more than 500 days of continuous and stable operation, demonstrated the reliability of the LAr detection technology at a scale never tested before. In this talk we will review the main characteristics and milestones of the construction and installation of protoDUNE-SP which provide a series of benchmarks for DUNE. The performance for several different detector working points will also be discussed.
\end{abstract}

40th International Conference on High Energy physics - ICHEP2020

July 28 - August 6, 2020

Prague, Czech Republic (virtual meeting)

${ }^{1}$ For the DUNE collaboration.

*Speaker 


\section{ProtoDUNE Single Phase at CERN}

ProtoDUNE Single Phase (ProtoDUNE-SP) is a large-dimension engineering prototype built at CERN, to validate the detector design for the first module of the DUNE far detector [1]. The detection technology is based on a Liquid Argon (LAr) Time Projection Chamber (TPC) where the charge is read by three planes of wires hosted in the Anode Plane Assemblies (APA). The primary scintillation light is read by the Photon Detection system which is installed in the APAs and implements three distinct technology designs, all based on light guide modules with wavelength shifter and readout by arrays of SiPMs. The TPC is made of two identical volumes: the 6 APAs (3 per side) are separated from a central cathode by a drift length of $3.6 \mathrm{~m}$. An electric field of $500 \mathrm{~V} / \mathrm{cm}$ (nominal value) is applied. The APA are instrumented with Cold Electronics (CE) to minimise the noise. Figure 1a shows the detector layout.

The construction of ProtoDUNE-SP started in September 2016 with the construction of the cryostat. In August 2018 the detector started to acquire data from a tagged particle beam, the $\mathrm{H} 4$ VLE [2]. After two months of beam data, the operation of ProtoDUNE-SP continued for about two years, recording cosmic ray events which allowed to extensively test the hardware and perform additional R\&D.

\section{Cryostat construction and detector assembly}

ProtoDUNE-SP implements, for the first time on a large scale particle physics detector, a new concept for very large dimension cryostats developed in the private sector. The design is based on the GTT membrane brevet [3] usually employed for transport and storage of Liquefied Natural Gas (Figure 1b) and re-engineered for particle physics applications and in particular LAr detectors. The technology foresees two layers of foam interleaved with plywood and, in contact with the LAr, a $1 \mathrm{~mm}$ stainless steel membrane with a corrugated shape which acts as a spring to absorb the material reaction to drastic temperature changes. An extensive validation campaign has been run to qualify the new cryostat concept in terms of tightness, stability and safety in agreement with both European and American standards. Thanks to the successful cryogenic operation of ProtoDUNE-SP, this design has been approved not only for the DUNE far detector modules but also for use in several other experiments requiring large dimension cryostats, like SBND or DarkSide. For the detector assembly, the various components arrived at the CERN experimental area in containers, shipped from the production sites. A dedicated clean room, built on one side of the cryostat, was used for assembly and testing of the detector modules (e.g. the test of the photon detection systems prior to their installation on the APAs). The clean room was equipped with a rail system to hang the large dimension detector components and move them easily either to the integrated cold test stand, the cold box, or move them into the cryostat, through the dedicated Temporary Construction Opening (TCO), for installation.

\section{ProtoDUNE-SP operations}

To achieve the goal of testing the detector response with beam particles, ProtoDUNE-SP has followed a very aggressive schedule. Nevertheless, the quality of the data recorded by the detector 

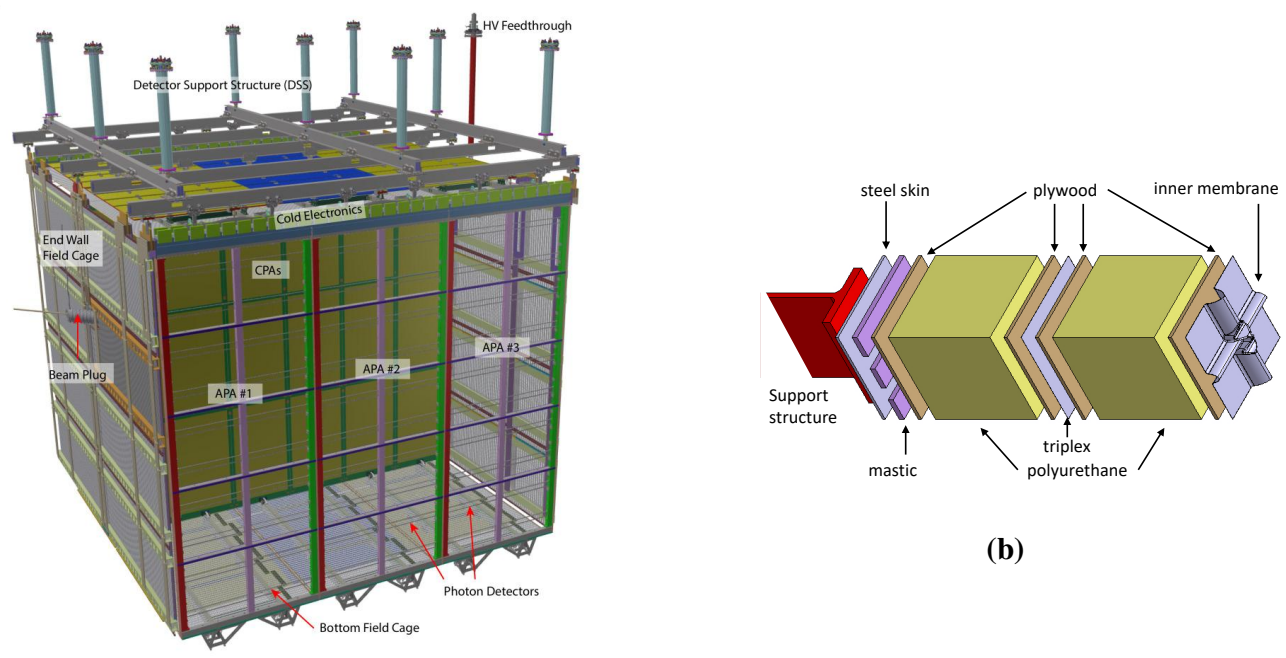

(b)

(a)

Figure 1: (a) The ProtoDUNE-SP detector layout. (b) The GTT cryostat inner vessel layers.

has been excellent since the very first day. It is worth noticing that the commissioning of the detector has been done at the same time as the first runs from the beam.

\subsection{Data taking with particle beams and cosmic rays}

To determine the capability of the detector to identify particles of different species and at different energies, data with hadrons and electrons have been recorded thanks to the CERN H4 beam line extension built explicitly for that purpose. The line provides hadron and electron beams from 0.3 to $7 \mathrm{GeV} / \mathrm{c}$. The sub- $\mathrm{GeV}$ momentum range $(0.5 \mathrm{GeV} / \mathrm{c}$ and $0.3 \mathrm{GeV} / \mathrm{c})$ beam is highly dominated by electrons due to the beam line length in which most of the pions decay before entering into the cryostat. About 4 million events have been recorded from the beamline in less than 2 months of data-taking. The CERN Long Shut Down 2 in November 2018 determined the end of the beam data but the activity of ProtoDUNE-SP continued, exploiting the large rate of cosmic rays crossing the detector due to its location on surface. Figure 2 shows a ProtoDUNE-SP event where a pion from the $2 \mathrm{GeV} / \mathrm{c}$ beam undergoes to a charge exchange interaction.

For the charge signals, careful pre-processing and calibration has been applied to the data to extract the ionisation signal, taking also into account the distortions derived by the space charge effect created by the large rate of cosmic rays interacting in the LAr. For the light signals, calibration and comparison of the three different technologies have been performed.

The detector has shown a very stable behaviour: the HV system has been reported stable $99.5 \%$ of the uptime, less than $1 \%$ of all APAs channels have been found noisy or dead. The purity of the liquid argon has also been excellent: thanks to the recirculation system implemented, impurities concentration has reached values less than $3.5 \mathrm{ppm} \mathrm{O}_{2}$ equivalent which corresponds to an electron lifetime better than $20 \mathrm{~ms}$. Finally, the photon detection system (PDS) has shown a very good time resolution of $14 \mathrm{~ns}$, good capabilities to match the ionisation signal, even in an environment busy as ProtoDUNE-SP, and calorimetric capabilities have also been reported. A summary of the main 


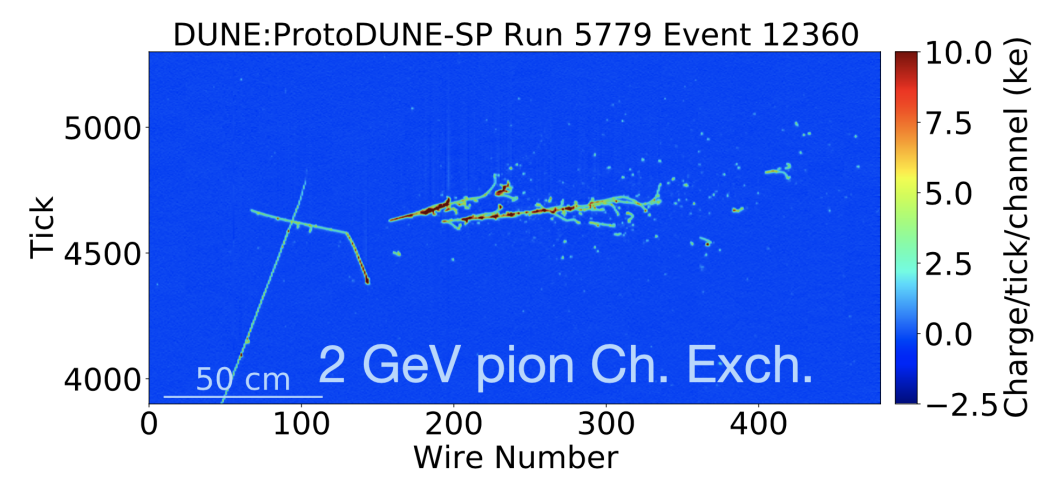

Figure 2: A $2 \mathrm{GeV} / \mathrm{c}$ pion charge exchange event recorded by protoDUNE-SP. The event shows, in the time vs wire space, the pion coming from the beam, entering from the left (starting around wire 70). The interaction occurs some tenth of $\mathrm{cm}$ later, producing a proton and a $\pi^{0}$ which decays on two photons. The showers from the two photons are clearly visible.

DUNE requirements and the ProtoDUNE-SP achievements are reported in Table 1. A detailed description of the ProtoDUNE-SP performance can be found in a recent publication [4].

Table 1: ProtoDUNE-SP performance for main parameters and corresponding DUNE specifications [4].

\begin{tabular}{|c|c|c|}
\hline Detector parameter & ProtoDUNE-SP performance & DUNE specification \\
\hline \multirow[t]{2}{*}{ Average drift electric field } & $500 \mathrm{~V} / \mathrm{cm}$ & $250 \mathrm{~V} / \mathrm{cm}(\mathrm{min})$ \\
\hline & & $500 \mathrm{~V} / \mathrm{cm}$ (nominal) \\
\hline LAr e-lifetime & $>20 \mathrm{~ms}$ & $>3 \mathrm{~ms}$ \\
\hline \multicolumn{3}{|l|}{ TPC+CE } \\
\hline Noise & (C) $550 \mathrm{e}$, (I) 650 e ENC (raw) & $<1000$ e ENC \\
\hline Signal-to-noise $\langle\mathrm{SNR}\rangle$ & (C) 48.7, (I) $21.2(\mathrm{w} / \mathrm{CNR})$ & \\
\hline CE dead channels & $0.2 \%$ & $<1 \%$ \\
\hline \multirow[t]{2}{*}{ PDS light yield } & 1.9 photons $/ \mathrm{MeV}$ & $>0.5$ photons $/ \mathrm{MeV}$ \\
\hline & (@ 3.3 m distance) & (@ cathode distance-3.6m) \\
\hline PDS time resolution & $14 \mathrm{~ns}$ & $<100 \mathrm{~ns}$ \\
\hline
\end{tabular}

\subsection{R\&D program}

ProtoDUNE-SP has served also as a test-bed for a number of R\&D projects, all aiming to provide useful measurements for the finalisation of the design for the first DUNE far detector. Some of those additional developments are summarised here:

- Xenon-doping: The liquid argon has been doped with a total of $13.5 \mathrm{~kg}$ of Xe which corresponds to about $20 \mathrm{ppm}$ in mass, injected in five steps. Xe shifts the wavelength of emitted scintillation light from $128 \mathrm{~nm}$, where LAr scintillates, to $175 \mathrm{~nm}$ where Xe scintillates. The main aims for this test are: (i) study the increase of the light yield and of the detection efficiency related to the longer attenuation length of Xe with respect to LAr; 
(ii) to study stability and uniformity of the response in a large detector, which has never been done before. To perform these tests, new PD systems sensitive to respectively LAr and Xe, only Xe and visible light have been installed in ProtoDUNE-SP. Preliminary results from this measurements look promising and a publication is in preparation.

- HV tests: The high voltage system has been pushed beyond its design specifications to check the margin of operations and enlarge the range of field-dependence studies (drift-velocity, space charge, ..). The voltage has been set to a $30 \%$ higher value than the nominal and the system has been found stable during the data acquisition which lasted a few days.

- DAQ developments: The data acquisition (DAQ) system also progressed greatly thanks to the ProtoDUNE-SP operations. The FELIX-based TPC readout $[5,6]$ has been validated and defined as baseline for the TPC readout in the first DUNE module. During the ProtoDUNESP operations, all APAs have been moved from the original RCE [7] to the FELIX readout. The FELIX readout has been tested and found stable also for large readout windows (up to 1s) with respect to the standard $3 \mathrm{~ms}$. Developments for a real time processing of the charge collected on the APA wires to identify Region-of-Interest for trigger decision ("hit finder") have shown promising results at the first test on the FELIX firmware, see Figure 3.

- Neutrons run: A deuterium-deuterium generator has been used to perform preliminary tests for the low energy calibration scheme foreseen for DUNE. The generator was set to deliver $10^{6}$ particles per second with an energy spectrum peaking at $2.5 \mathrm{MeV}$ and it has been positioned on the top of one ProtoDUNE-SP spare penetrations on the roof. The detection of low energy gammas $(6.1 \mathrm{MeV})$ coming from the neutron capture is the expected signature for neutron interactions with LAr. The analysis of the total charge collected by the APA wires shows first hints of the detection of those neutrons. Similarly, the real-time processing of those data by the FELIX hit finder shows a clear increase of low-energy deposits compared to the common cosmic ray runs. Finally, a third analysis based on semantic segmentation done by means of a neural network shows promising results on the identification of those low energy deposits and the understanding of the propagation of the neutrons in the TPC volume.

\subsection{Conclusions}

ProtoDUNE-SP has been turned off on July 2020, after two years of operations. The detector has served as an engineering prototype for the cryostat design and the cryogenic system and as a test bed for the detector assembly procedure. ProtoDUNE-SP has provided data of excellent quality both coming from tagged beam particles (hadrons, electrons) and cosmic rays. With these data the detector performance has been estimated and found to largely fulfil the DUNE requirements. A recent publication summarises these achievements [4]. Physics measurements are in preparation.

To conclude, ProtoDUNE-SP has served to fully validate and characterise the design for the first module of the DUNE far detector and also to perform additional R\&D, both in hardware and DAQ towards DUNE. 


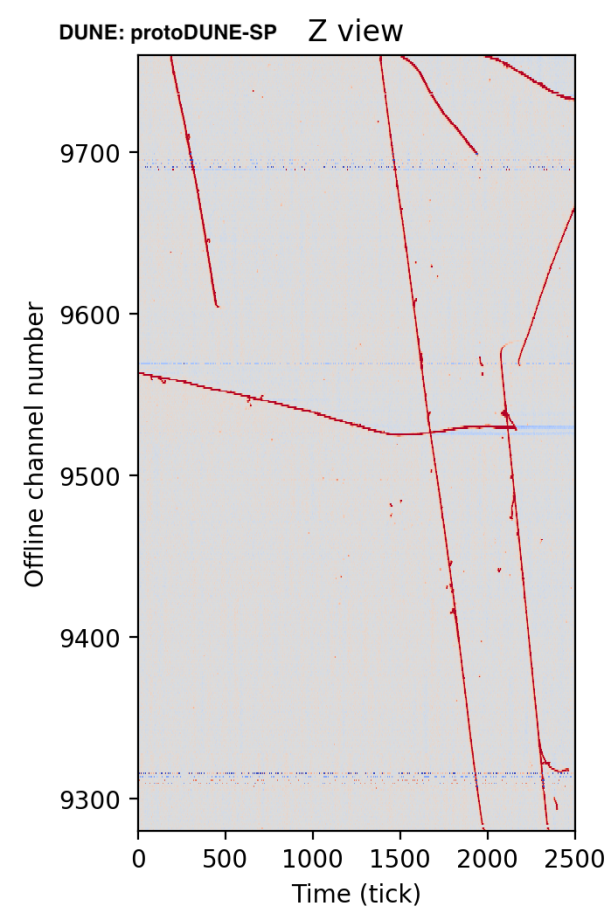

(a)

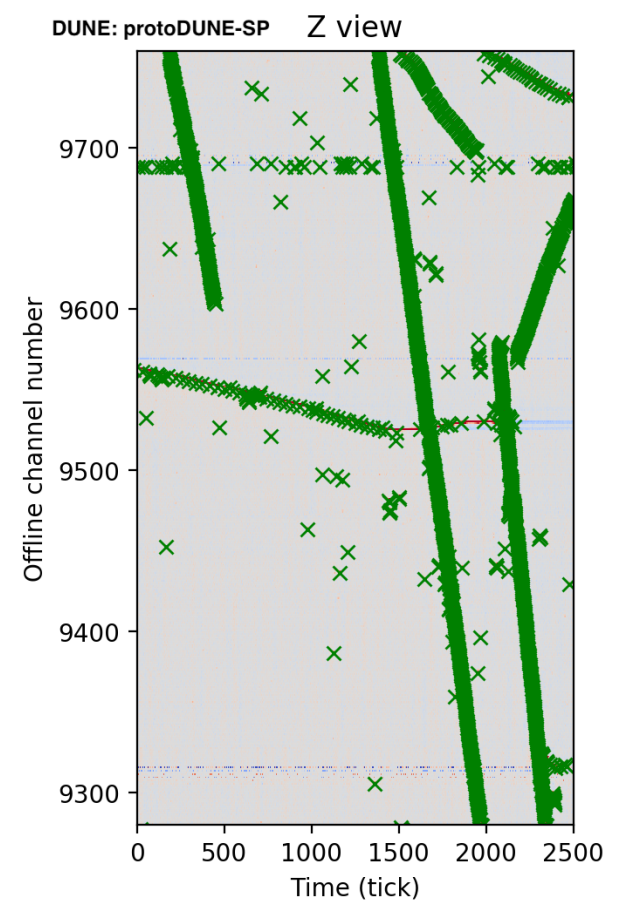

(b)

Figure 3: Example of the FELIX online hit-finder for one of the three wire planes (collection plane). (a) Event display obtained from the standard ProtoDUNE-SP data processing and (b) the hits found by FELIX.

\section{References}

[1] B. Abi, et al., (The DUNE collaboration), The Dune Deep Underground Neutrino Experiment (DUNE), Far Detector Technical Design Report, Volume I: Introduction to DUNE, arXiv:2002.02967.

[2] A. C. Booth, N.Charitonidis, et al, Particle production, transport and identification in the regime of 1 - 7 GeV/c, Phys. Rev. Accel. Beams 22, 061003 (2019).

[3] GTT Membrane Technologies, http://www.gtt-training.co.uk/gtt-membrane-technologies.

[4] B. Abi, et al, (The DUNE collaboration), First results on ProtoDUNE-SP liquid argon time projection chamber performance from a beam test at the CERN Neutrino Platform, arXiv:2007.06722.

[5] Anderson, J. et al., FELIX: a PCIe based high-throughput approach for interfacing front-end and trigger electronics in the Upgrade framework, JINST 11 (2016) 12, C12023.

[6] Borga, Andrea et al, FELIX based readout of the Single-Phase ProtoDUNE detector, arXiv 1806.09194, IEEE Real-Time 2018 conference proceedings. 
[7] R. Herbst et al., Design of the SLAC RCE Platform: A general purpose ATCA based data acquisition system, 2014 IEEE Nuclear Science Symposium and Medical Imaging Conference (NSS/MIC), Seattle, WA, 2014, pp. 1-4. 